\title{
Efecto de la aplicación de abonos foliares y enmiendas orgánicas, sobre el rendimiento de repollo corazón de buey (Brassica oleracea L.), en Chachapoyas, Amazonas
}

Effect of the application of foliar fertilisers and organic amendments, on the performance of ox heart cabbage (Brassica oleracea L.), in Chachapoyas, Amazonas

Roicer Collazos Silva ${ }^{1}$, Jessy Patricia Arista Bustamante ${ }^{1}$, Silvia Karina Oyarce ${ }^{1}$ y Eyner Huamán Huamán ${ }^{1}$

\section{RESUMEN}

La presente investigación tuvo como objetivo evaluar la respuesta productiva del repollo corazón de buey (Brassica oleracea L.) a la aplicación de diferentes dosis foliares y enmiendas orgánicas. Para ello se instaló un experimento con 12 tratamientos en los cuales se combinaron dos tipos de enmiendas orgánicas (guano de isla y humus de lombriz), y tres dosis de fertilizante foliar de fórmula 20-20-20. Se evaluaron las variables altura de planta (cm), peso del corazón $(\mathrm{g})$, diámetro de copa $(\mathrm{cm})$, diámetro de corazón $(\mathrm{cm})$ y peso de la planta $(\mathrm{g})$ en 10 plantas por unidad experimental tres meses después de establecer el experimento, obteniéndose como resultado que el tratamiento compuesto por guano de isla en una dosis de $7 \mathrm{tn} / \mathrm{ha}$ más tres aplicaciones de abono foliar en una dosis de $2 \mathrm{~L} / 200 \mathrm{~L}$ de agua, fue superior a los demás tratamientos en las variables peso del corazón, diámetro del corazón y peso total de la planta con 1091,6 g, 45,9 cm y 1868,6 g respectivamente, lográndose incrementar hasta en un 57,2\% en rendimiento. Por esta razón, la adición de abonos foliares a los planes de fertilización, mejoran los rendimientos del cultivo, sin embargo hay que tener en cuenta que su incorporación genera mayores gastos e incrementa los costos de producción.

Palabras claves: Guano de isla, humus, foliar, enmienda orgánica, repollo.

\begin{abstract}
The goal of this research was to evaluate the productive response of ox heart cabbage (Brassica oleracea L.) to the application of different foliar doses and organic amendments. For it, an experiment was installed with 12 treatments in which two types of organic amendments were combined (island guano and worm humus), and three doses of foliar fertilizer of formula 20-20-20. The variables plant height $(\mathrm{cm})$, heart weight $(\mathrm{g})$, canopy diameter $(\mathrm{cm})$, heart diameter $(\mathrm{cm})$ and plant weight $(\mathrm{g})$ were evaluated in 10 plants per experimental unit three months after establishing the experiment, obtaining as a result that the treatment composed of island guano in a dose of $7 \mathrm{tn} / \mathrm{ha}$ plus three applications of foliar fertilizer in a dose of $2 \mathrm{~L} / 200 \mathrm{~L}$ of water, was superior to the other treatments in the variables weight of the heart, diameter of the heart and total weight of the plant, with $1091.6 \mathrm{~g}, 45.9 \mathrm{~cm}$ and $1868.6 \mathrm{~g}$, respectively, being able to increase up to $57.2 \%$ in yield. For this reason, the addition of foliar fertilizers to fertilization plans improves crop yields, however, it must be taken into account that their incorporation generates higher costs and increases production expenses.
\end{abstract}

Keywords: Island guano, humus, foliar, organic amendment, cabbage.

\footnotetext{
${ }^{1}$ Universidad Nacional Toribio Rodríguez de Mendoza de Amazonas (UNTRM-A), Instituto de Investigación para el Desarrollo Sustentable de Ceja de Selva, Calle Higos Urco N³42-350-356, Calle Universitaria N 304, Chachapoyas, Perú

"Autor de correspondencia. E-mail: rcollazos@indes-ces.edu.pe
} 


\section{INTRODUCCIÓN}

El repollo corazón de buey (Brassica oleracea L. var. capitata), es una hortaliza cuyo cultivo puede presentar una fase vegetativa, precoz, intermedia o tardía, la cual está influenciada por las condiciones ambientales, principalmente temperatura y altitud (FHIA, 2012).

Tiene un requerimiento nutricional de $120 \mathrm{~kg} / \mathrm{ha}$ de Nitrógeno, $45 \mathrm{~kg} /$ ha de $\mathrm{P}_{2} \mathrm{O}_{5}, 160 \mathrm{~kg} / \mathrm{ha}$ de $\mathrm{K}_{2} \mathrm{O}, 100$ $\mathrm{kg} / \mathrm{ha}$ de Ca y $6 \mathrm{~kg} / \mathrm{ha}$ de $\mathrm{Mg}$ para obtener un rendimiento de 35 tn/ha. En el año 2010, a nivel mundial se registró una cosecha de 2,08 millones de hectáreas, una producción de 57,96 millones de toneladas, con un rendimiento promedio anual de 27,81 Tn/Ha (Guerrero, 1998).

La respuesta de la planta a las adiciones de nutrientes depende de la especie, la forma en que se aplique el fertilizante, su concentración y frecuencia de aplicación, así como la fase de crecimiento de la planta (Kuepper, 2003). Necesitan nutrientes inorgánicos, además de dióxido de carbono y agua para su crecimiento y producción (Kannan et al., 2009). Desde tiempo atrás la calidad del suelo está siendo dañada debido a prácticas agrícolas agresivas y la abundancia de contaminantes orgánicos e inorgánicos. Este estado de fertilidad del suelo ha llevado a la búsqueda de fuentes nutricionales que pueden restaurar condiciones más saludables (Vargas et al., 2008).

Las enmiendas orgánicas mejoran las condiciones del suelo en cuanto a carbono orgánico, estabilidad estructural y conductividad hidráulica (Rotondo et al., 2009). La incorporación de materia orgánica fresca al suelo puede presentar varias desventajas, como la inestabilidad del material, presencia de contaminantes y la acción de microorganismos fitopatógenos. Ante esto los procesos de compostaje disminuyen e, inclusive, eliminan ciertos peligros, además de proporcionar materia orgánica con mejores propiedades que favorecen su aplicación y mejoran sus propiedades físicas (Vargas et al., 2008). Aun así necesita un período de tiempo para mineralizar y suministrar los nutrientes necesarios a los cultivos además de necesitar una gran cantidad de producto para compensar los requerimien- tos nutricionales de los cultivos (Tejada y González, 2006).

Se deben conocer las exigencias nutricionales de un cultivo, además de la cantidad e intensidad de nutrientes que estén contenidos en el suelo y cuando este no pueda suplir adecuadamente estos requerimientos para un normal desarrollo de las plantas, es necesario su adición en cantidades y formas adecuadas (Salas, 2002).

La fertilización foliar considerada como una agrotécnica (Alshaal et al., 2017), permite el uso más óptimo de algunos fertilizantes ante el creciente costo que se ha generado en la actualidad para la producción de los mismos (Kannan et al., 2009). Se trata de una herramienta significativa para el manejo sostenible y productivo de los cultivos (Fernández et al., 2015), y es utilizado como medio para proporcionar cantidades suplementarias de nutrientes, hormonas vegetales, estimulantes y otras sustancias beneficiosas (Kuepper, 2003). Se utiliza mediante procesos en los cuales se aplica una solución con nutrientes al follaje, lo cual engloba contacto con la hoja y adsorción a la superficie de la misma, penetración cuticular/estomática a través de otras estructuras epidérmicas, absorción celular y penetración en los compartimentos celulares metabólicamente activos en la hoja. Finalmente, la translocación y la utilización de los nutrientes absorbidos por la planta (Fernández et al., 2015). Los efectos obtenidos de esta fertilización foliar son aumentos en el rendimiento, resistencia a enfermedades y plagas de insectos, tolerancia a la sequía y mejor calidad de los cultivos (Kuepper, 2003). Sin embargo, esta no sustituye la fertilización del suelo, y constituye una práctica sugerente para complementar la nutrición edáfica y para sustituir ciertos nutrientes durante períodos críticos del cultivo o de alta demanda nutricional, como la floración y el llenado de granos y frutos (Molina, 2002). Asimismo ayuda a las plantas en la recuperación del choque de trasplante, daño por granizo, climas extremos (Kuepper, 2003), así como en períodos de estrés causados por sequías, encharcamientos, heladas, aplicación de agroquímicos. Por último, previene 
en casos en que las condiciones de suelos limiten el crecimiento y función de las raíces, como el drenaje, toxicidad de aluminio, salinidad, etc., que afectan la absorción radical de nutrimentos (Molina, 2002). Sin embargo se debe tener en cuenta que a mayor edad de las hojas, disminuye su capacidad de absorción, debido a un aumento en la permeabilidad de la membrana, a una disminución en la actividad metabólica, y a un incremento en el grosor de la cutícula (Salas, 2002).

Algunos indican que las plantas bien nutridas tienen una mejor capacidad para resistir plagas y enfermedades. Por otro lado hay quienes niegan cualquier relación entre la nutrición de los cultivos y la ocurrencia de plagas (Kannan et al., 2009). La respuesta de las plantas a la fertilización foliar varía entre especies y cultivares y también depende de la fenología de la planta, de su estado fisiológico y del medio ambiente en donde esta crece (Fernández et al., 2015).

La fertilización foliar es de manera teórica más amigable con el ambiente en comparación con la aplicación de nutrientes por la vía radicular, ya que tiene una incidencia más inmediata y encaminada al objetivo. Además, producto de esta comparativa con la fertilización del suelo, los nutrientes son aplicados de manera directa en los tejidos vegetales y en las etapas críticas del desarrollo de las plantas (Fernández et al., 2015). En términos de absorción de nutrientes, la fertilización foliar puede ser de 8 a 20 veces más eficiente que la aplicación en el suelo, sin embargo, esta eficacia no siempre se logra en la práctica (Kuepper, 2003).

La presente investigación tiene por objetivo conocer la respuesta que tiene el repollo corazón de buey (Brassica oleracea L.) en la aplicación de diferentes dosis foliares de Fertiphos 20-20-20 y enmiendas orgánicas para un total de 12 tratamientos.

\section{MATERIALY MÉTODOS}

\section{Ubicación del experimento}

La investigación se instaló en el área de la estación experimental Chachapoyas del Instituto de Investigación para el Desarrollo Sustentable de Ceja de Selva sector INIA, a una altitud aproximada de 2300 m.s.n.m. (Figura 1).

\section{Diseño del experimento}

Se utilizó un diseño completamente aleatorizado (DCA), con 12 tratamientos y 3 repeticiones, siendo los tratamientos los que se describen en la Tabla 1 .

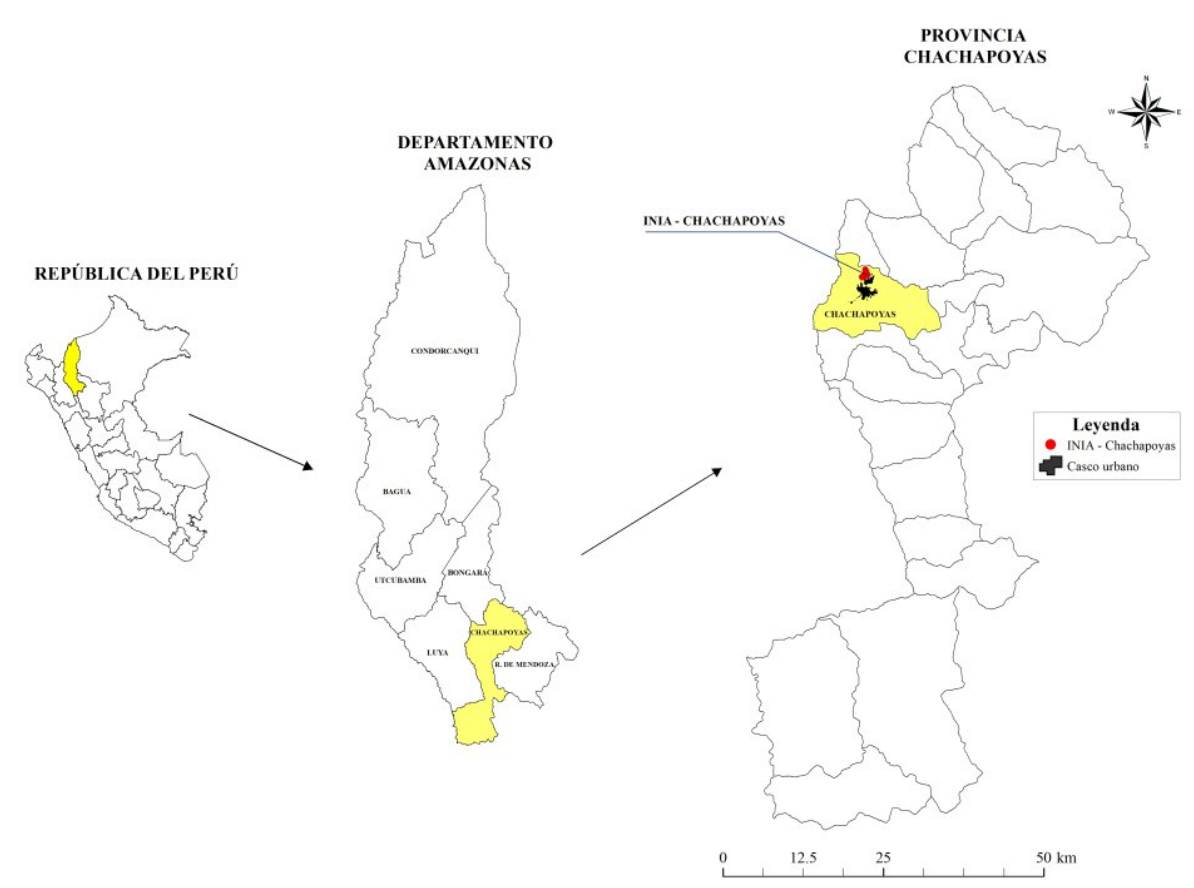

Figura 1. Mapa de ubicación del sector INIA en la ciudad de Chachapoyas 
Tabla 1. Descripción de los 12 tratamientos empleados

\begin{tabular}{ccc}
\hline Tratamiento & Enmienda & Aplicación foliar \\
\hline T1 & Sinenmienda & Sin ab \\
T2 & Guano isla* & Sin ab \\
T3 & Humus lombriz* & Sin ab \\
T4 & Sin enmienda & ab $1,5 \mathrm{~L} / 200 \mathrm{LH}_{2} \mathrm{O}$ \\
T5 & Guano isla* & ab $1,5 \mathrm{~L} / 200 \mathrm{LH}_{2} \mathrm{O}$ \\
T6 & Humus lombriz* & ab $1,5 \mathrm{~L} / 200 \mathrm{LH}_{2} \mathrm{O}$ \\
T7 & Sin enmienda & ab 2L/200 $\mathrm{LH}_{2} \mathrm{O}$ \\
T8 & Guano isla* & ab 2L/200 $\mathrm{LH}_{2} \mathrm{O}$ \\
T9 & Humus lombriz* & ab 2L/200 $\mathrm{LH}_{2} \mathrm{O}$ \\
T10 & Sin enmienda & ab 2,5L/200 $\mathrm{LH}_{2} \mathrm{O}$ \\
T11 & Guano isla* & ab 2,5L/200 $\mathrm{LH}_{2} \mathrm{O}$ \\
T12 & Humus lombriz* & ab 2,5L/200 $\mathrm{LH}_{2} \mathrm{O}$ \\
\hline
\end{tabular}

$*=7 \mathrm{tn} / \mathrm{ha} ; \mathrm{ab}=$ abono foliar; $\mathrm{H} 2 \mathrm{O}=$ agua

El área total del experimento fue de $216 \mathrm{~m} 2$, donde las unidades experimentales tuvieron una área de 4,8 $\mathrm{m} 2$, sembrándose 36 plantas por unidad experimental, y evaluándose 10 plantas por unidad.

\section{Manejo del experimento}

Para el manejo del experimento, en primer lugar, se seleccionó el terreno. Este evidenció una topografía plana con características edáficas adecuadas para el cultivo de repollo (Tabla 2). El análisis del suelo se realizó en el Laboratorio de Investigación en Suelos y Aguas (LABISAG) de la Universidad Nacional Toribio Rodríguez de Mendoza(UNTRM).

Tabla 2. Resultados de análisis de suelo

\begin{tabular}{cc}
\hline Clase textural & Arcilla \\
C.E.(1:1) & $0,47 \mathrm{~ms} / \mathrm{cm}$ \\
pH & 7,32 \\
K & $170,01 \mathrm{ppm}$ \\
P & $5,32 \mathrm{ppm}$ \\
M. O. & $4,14 \%$ \\
C & $2,4 \%$ \\
N & $0,21 \%$ \\
\hline
\end{tabular}

La labranza del terreno se hizo en dos etapas; en la primera se hizo un roturado de terreno mediante tracción mecánica, y posteriormente se hizo un mullido utilizando herramientas de labranza.

Las siembra se realizó con una densidad de 62500 plantas/ha. La aplicación de las enmiendas se hizo en el momento de preparar el terreno. Las aplicaciones de abono foliar se realizaron a los 15, 30, 45 y 60 días después de la siembra.

Se evaluaron 10 plantas por unidad experimental a los tres meses de establecido el experimento, y se evalua- ron las variables altura de planta $(\mathrm{cm})$, diámetro de copa $(\mathrm{cm})$, peso total de la planta $(\mathrm{g})$, peso del corazón (gr) y diámetro de corazón $(\mathrm{cm})$.

Asimismo, para el control de plagas y enfermedades se hizo el monitoreo y evaluación de plagas claves en la zona: polilla dorzo diamane o plutela (Plutela xilostela), pulgón (Macrosiphum sp.), pieris (Pieris rapae monuste) y diabróticas (Diabrotica speciosa). Estos fueron controlados integrando métodos culturales, mecánicos, etológicos y aplicación de productos químicos, evitando que el nivel de daño supere los niveles críticos por cada plaga.

\section{Procesamiento de datos y análisis estadístico}

El procesamiento de datos se realizó utilizando el software estadístico STATISTIX 8, además como programa complementario de manejo y rellenado de datos se utilizó Microsoft Excel 2013.

\section{RESULTADOS}

\section{Altura de planta}

Los resultados de esta variable fueron sometidos a la prueba Duncan, cuyos resultados según se muestra en la Tabla 3, mostraron diferencias significativas entre tratamientos.

Tabla 3. Prueba de Duncan para la variable altura de planta

\begin{tabular}{ccc}
\hline Tratamientos & $\begin{array}{c}\text { Alturade } \\
\text { planta }(\mathbf{c m})\end{array}$ & $\begin{array}{c}\text { Prueba de } \\
\text { Duncan }\end{array}$ \\
\hline T1 & 36,6 & $\mathrm{AB}$ \\
T2 & 35,8 & $\mathrm{AB}$ \\
T3 & 35,95 & $\mathrm{AB}$ \\
T4 & 36,35 & $\mathrm{AB}$ \\
T5 & 38,45 & $\mathrm{AB}$ \\
T6 & 32,95 & $\mathrm{~B}$ \\
T7 & 41,7 & $\mathrm{~A}$ \\
T8 & 38,55 & $\mathrm{AB}$ \\
T9 & 32,15 & $\mathrm{~B}$ \\
T10 & 39,9 & $\mathrm{~A}$ \\
T11 & 40,15 & $\mathrm{~A}$ \\
T12 & 35,1 & $\mathrm{AB}$ \\
\hline
\end{tabular}

Letras iguales (A y B) no muestran diferencias significativas $(\mathrm{p}<0,05)$

Asimismo tal como se puede apreciar en la Figura 2, los tratamientos T7, T10 y T11 obtuvieron los mayores promedios de altura con $41,7 \mathrm{~cm}, 39,9 \mathrm{~cm}$ y $40,15 \mathrm{~cm}$, respectivamente; mientras que el T9 obtuvo el menor promedio de altura alcanzando $32,15 \mathrm{~cm}$. 


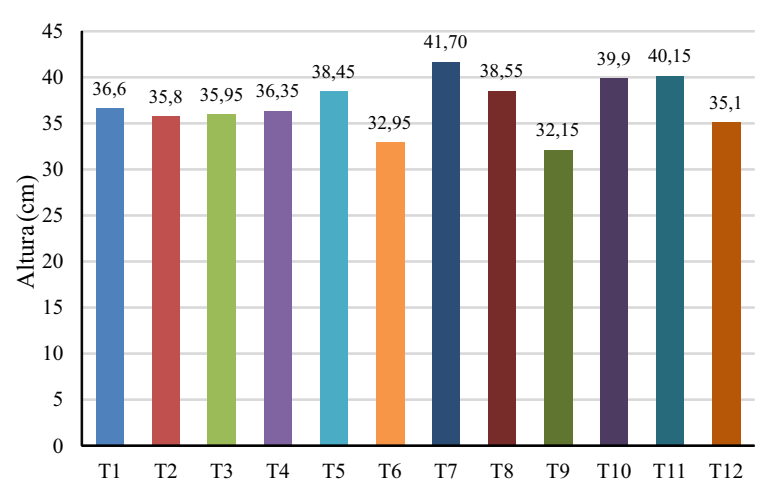

Figura 2. Altura promedio de planta por tratamiento.

\section{Diámetro de copa}

Los resultados de esta variable fueron sometidos a la prueba Duncan, cuyos resultados se muestran en la Tabla 4, mostrando diferencias significativas entre tratamientos

Tabla 4. Prueba de Duncan para la variable diámetro de copa entre tratamientos

\begin{tabular}{ccc}
\hline Tratamientos & $\begin{array}{c}\text { Diámetro } \\
\text { copa }(\mathbf{c m})\end{array}$ & $\begin{array}{c}\text { Prueba de } \\
\text { Duncan }\end{array}$ \\
\hline T1 & 44,55 & EF \\
T2 & 52,9 & A \\
T3 & 46,95 & CDE \\
T4 & 45,525 & DEF \\
T5 & 47,375 & BCDE \\
T6 & 40,3 & G \\
T7 & 50,65 & ABC \\
T8 & 48,775 & BCD \\
T9 & 42 & FG \\
T10 & 50,8 & AB \\
T11 & 49,25 & ABC \\
T12 & 42,95 & FG \\
\hline
\end{tabular}

Letras iguales (Ay B) no muestran diferencias significativas $(\mathrm{p}<0,05)$

Tal y como se puede apreciar en la Figura 3, los tratamientos T2 y T10 son los que obtuvieron los mejores resultados en cuanto a diámetro de copa, alcanzado 18 y $12,8 \mathrm{~cm}$, respectivamente. Por el contrario, el tratamiento $\mathrm{T} 6$ obtuvo el menor resultado, con $40,3 \mathrm{~cm}$

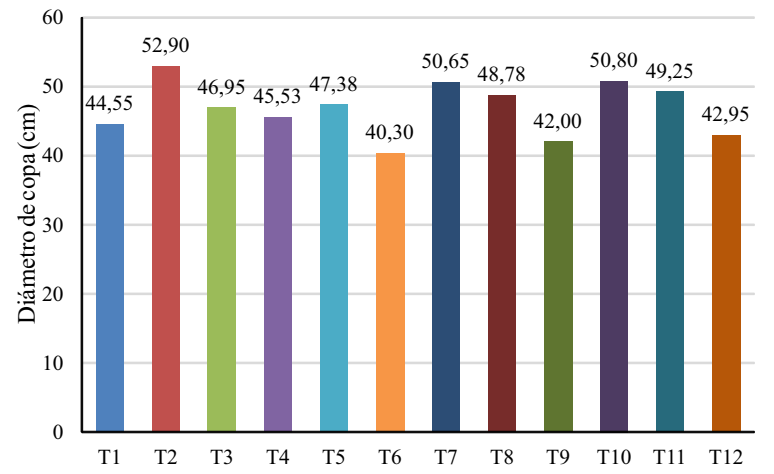

Figura 3. Diámetro de copa por tratamiento.

\section{Peso total de la planta}

Los resultados de esta variable fueron sometidos a la prueba Duncan, cuyos resultados según muestra la Tabla 5, evidenciaron diferencias significativas entre tratamientos.

Tabla 5. Prueba de Duncan para la variable peso total de la planta entre tratamientos

\begin{tabular}{ccc}
\hline Tratamientos & $\begin{array}{c}\text { Peso total dela } \\
\text { planta }(\mathbf{g})\end{array}$ & $\begin{array}{c}\text { Prueba de } \\
\text { Duncan }\end{array}$ \\
\hline T1 & 1309,1 & BCD \\
T2 & 1681,8 & AB \\
T3 & 1238,8 & BCD \\
T4 & 1411,5 & ABCD \\
T5 & 1868,6 & A \\
T6 & 1077,3 & CD \\
T7 & 1548,9 & ABC \\
T8 & 1636,5 & AB \\
T9 & 1034,4 & D \\
T10 & 1429,0 & ABCD \\
T11 & 1437,2 & ABCD \\
T12 & 1415,9 & ABCD \\
\hline
\end{tabular}

Letrasiguales (AyB) no muestran diferencias significativas $(p<0,05)$

De nuevo, tal y como puede apreciarse en la Figura 4, el tratamiento T5 registró el mayor peso total, con 1868,6 g, mientras que el T9 registró el menor peso con 1034,4 g.

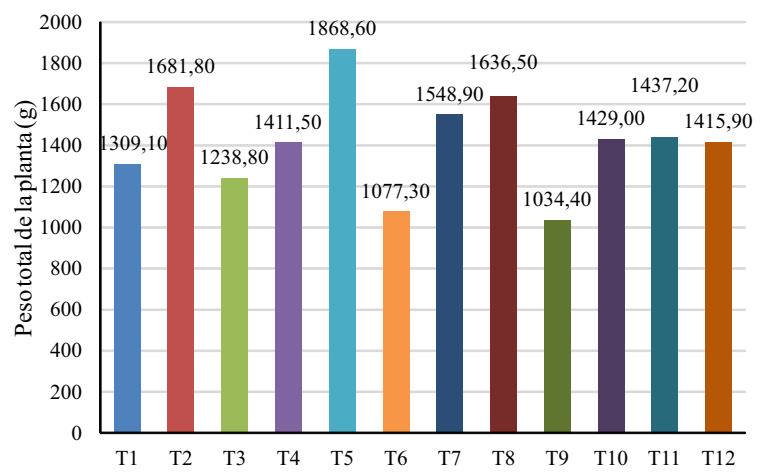

Figura 4. Peso total de la por tratamiento.

\section{Peso del corazón}

De igual forma los resultados de esta variable fueron sometidos a la prueba Duncan, cuyos resultados según muestra la Tabla 6, mostraron diferencias significativas entre tratamientos. 
Tabla 6. Prueba de Duncan para la variable peso del corazón entre tratamientos

\begin{tabular}{ccc}
\hline Tratamientos & $\begin{array}{c}\text { Peso del } \\
\text { corazón }(\mathbf{g})\end{array}$ & $\begin{array}{c}\text { Prueba de } \\
\text { Duncan }\end{array}$ \\
\hline T1 & 694,1 & $\mathrm{BC}$ \\
T2 & 795,1 & $\mathrm{BC}$ \\
T3 & 780,2 & $\mathrm{BC}$ \\
T4 & 766,5 & $\mathrm{BC}$ \\
T5 & 1091,6 & $\mathrm{~A}$ \\
T6 & 612,1 & $\mathrm{C}$ \\
T7 & 807,7 & $\mathrm{BC}$ \\
T8 & 940,95 & $\mathrm{AB}$ \\
T9 & 562,95 & $\mathrm{C}$ \\
T10 & 747,3 & $\mathrm{BC}$ \\
T11 & 681,6 & $\mathrm{BC}$ \\
T12 & 802,45 & $\mathrm{BC}$ \\
\hline
\end{tabular}

Letras iguales (Ay B) no muestran diferencias significativas $(\mathrm{p}<0,05)$

Asimismo, como se puede apreciar en la Figura 5, el tratamiento $\mathrm{T} 5$ registró el mayor peso de corazón, con 1091,6 g, seguido del tratamiento T8. Mientras que los tratamientos $\mathrm{T} 6$ y $\mathrm{T} 9$ registraron los menores valores, con 612,1 gy $562,95 \mathrm{~g}$, respectivamente.

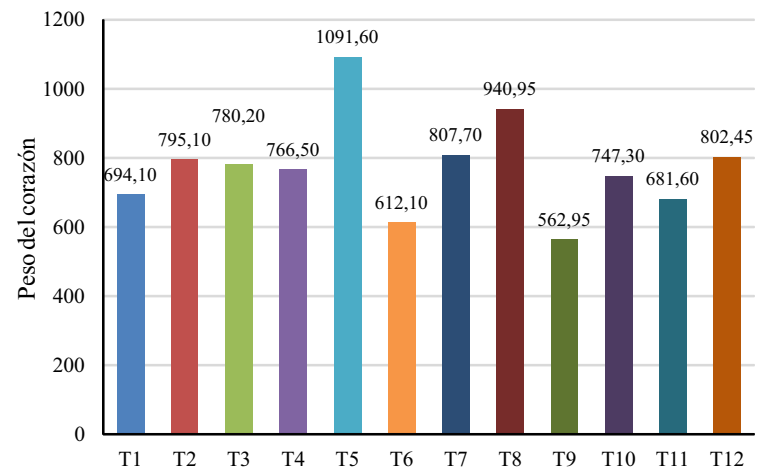

Figura 5. Peso total de la planta por tratamientos.

\section{Diámetro de corazón}

Los resultados de esta variable fueron sometidos a la prueba Duncan, cuyos resultados según muestra la Tabla 7, exhibieron diferencias significativas entre tratamientos

Tal y como se puede apreciar en la Figura 6, los tratamiento T5, T7, T8 y T2 obtuvieron los mejores resultados, con $14,6 \mathrm{~cm}, 14,2 \mathrm{~cm}, 14,1 \mathrm{~cm}$ y $14,0 \mathrm{~cm}$, respectivamente. Por el contrario, el T6 obtuvo el menor promedio con $12,05 \mathrm{~cm}$.
Tabla 7. Prueba de Duncan para la variable diámetro de corazón entre tratamientos

\begin{tabular}{ccc}
\hline Tratamientos & $\begin{array}{c}\text { Diámetrode } \\
\text { corazón }(\mathbf{c m})\end{array}$ & $\begin{array}{c}\text { Prueba de } \\
\text { Duncan }\end{array}$ \\
\hline T1 & 13,55 & $\mathrm{ABC}$ \\
T2 & 14 & $\mathrm{ABC}$ \\
T3 & 13,5 & $\mathrm{ABC}$ \\
T4 & 13,15 & $\mathrm{ABC}$ \\
T5 & 14,6 & $\mathrm{~A}$ \\
T6 & 12,05 & $\mathrm{C}$ \\
T7 & 14,2 & $\mathrm{AB}$ \\
T8 & 14,1 & $\mathrm{ABC}$ \\
T9 & 12,5 & $\mathrm{BC}$ \\
T10 & 13,7 & $\mathrm{ABC}$ \\
T11 & 13,45 & $\mathrm{ABC}$ \\
T12 & 13 & $\mathrm{ABC}$ \\
\hline
\end{tabular}

Letras iguales (Ay B) no muestran diferencias significativas $(\mathrm{p}<0,05)$

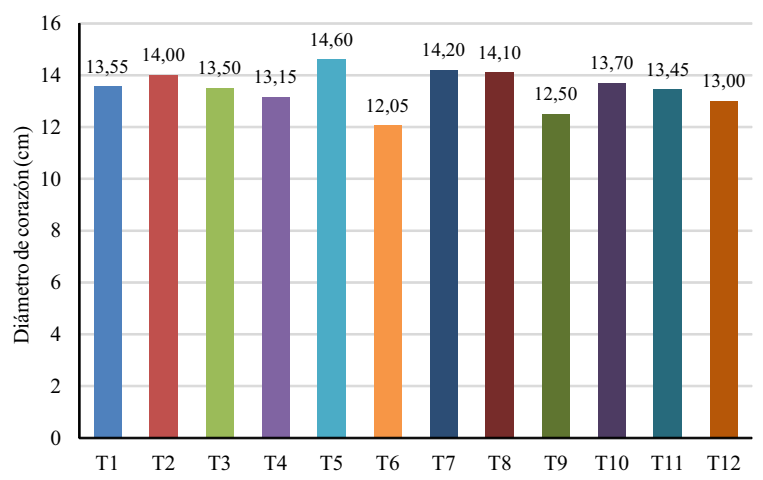

Figura 6. Diámetro de copa por tratamientos.

\section{IV.DISCUSIÓN}

De los resultados obtenidos, la adición de guano de isla obtuvo mejores resultados en el desarrollo y producción del repollo corazón de buey (Brassica oleracea), notándose que las variables diámetro de copa, peso de planta, diámetro del corazón y peso del corazón, son mayores al testigo. En la aplicación de humus de lombriz, estos resultados coinciden con los resultados encontrados por Bellido (2010), quien evaluó el efecto de niveles de aplicación de guano de isla en el cultivo de tomate, encontrando que niveles crecientes de guano de isla se traduce en mejores rendimientos del cultivo. Así mismo se observó que la aplicación de guano de isla más la aplicación de abonos foliares, tuvo un efecto positivo evidenciándose que a medida que se incrementa la dosis de abono foliar el rendimiento se incrementa. Dicho resultado es acorde con Fernandez et al. (2015), quienes mencionan que es factible alimentar las plantas por vía foliar, en particular cuando 
se trata de corregir deficiencias de elementos menores. Sin embargo, para elementos mayores estos mismos autores mencionan que la nutrición foliar solamente puede complementar, y en ningún caso substituir, a la fertilización del suelo, debiéndose a que las dosis de aplicación que pueden administrarse por vía foliar son muy pequeñas en relación con los niveles de fertilización requeridos por los cultivos para alcanzar altos niveles de productividad.

Por otro lado el mayor promedio de altura alcanzado fue de 41,7 cm, que se logró con la sola adición de abono foliar en una dosis de $200 \mathrm{ml}$. Estos resultados superaron a los obtenido por Morales (2010), quien evaluó la fertilización foliar orgánica e inorgánica aplicada al cultivo de repollo en la variedad Copenhague market, en donde obtuvo que con la aplicación de Fertidrip (12-45-12: 35gr/7,5lts de agua) se dieron los mejores datos en cuanto a mayor altura, alcanzando $16,82 \mathrm{~cm}$. Cabe mencionar que las diferencias con respecto a la variedad evaluada en cada investigación pueden ser el factor que incida en los resultados encontrados.

Nina (2014), evaluó el efecto del abonamiento en cuatro variedades de repollo con dos tipos de compost. En dicha investigación obtuvo un diámetro de corazón, en la variedad corazón de buey, de $24,30 \mathrm{~cm}$, en la cual aplicó compost (estiércol de vacuno, forraje verde de alfalfa y hojas de repollo) más la adición de microorganismos eficientes. Estos resultados fueron superiores a los obtenidos en la presente investigación ya que el diámetro de corazón alcanzó el máximo promedio de $14,6 \mathrm{~cm}$, tratamiento al cual se adicionó fertilizante foliar Fertiphos 20-20-20 en una dosis de 1,5 litros/200L y guano de guano de isla. Al respecto, Fernández et al. (2015) señala que una combinación de tratamientos de fertilizantes foliares y al suelo pueden ayudar a aumentar la eficiencia de absorción de nutrientes y limitar la polución del suelo, particularmente con elementos tales como N y/o P. Mientras que para el diámetro de copa el mayor valor obtenido fue de $18 \mathrm{~cm}$ con la sola adición de $10 \mathrm{~s} 7 \mathrm{~kg} /$ parcela de guano de isla. Zeledón(2014) evaluó el efecto de tres métodos de fertilización orgánica en la producción de dos variedades de repollo, en donde obtuvo un diámetro promedio de 39,63 (variedad Izalco) y 40,53 (variedad Bravo) con la aplicación de tratamiento edáfico/foliar de $105,2 \mathrm{lb}$ de bioperla y 3,2L de Supermagro por tratamiento.

Matus (1999) obtuvo resultados de peso de cabeza entre 1,2 y $1,35 \mathrm{~kg}$, en el cual se evaluaron tres distancias $(40,50$ y $660 \mathrm{~cm})$ y dos dosis de fertilizante $(18$ 46-0) (5,7 qq/Ha y 8,5 qq/Ha) en la variedad repollo híbrido Izalco. Armas (2013), evaluó el efecto de niveles de compost con fertilizante orgánico foliar sobre el repollo. Este autor obtuvo un promedio de peso de corazón de 1,25 kg con la aplicación de $8 \mathrm{~kg} / \mathrm{m}^{2}$ de compost y DUAOF. En la presente investigación se obtuvieron resultados más bajos ya que el mayor peso promedio de corazón fue de $1,09 \mathrm{~kg}$ en el cual se aplicaron $150 \mathrm{ml}$ de fertilizante foliar Fertiphos 20-20-20 y 7 $\mathrm{Tn} / \mathrm{Ha}$ de guano de isla. Ante ello Kuepper (2003), menciona que la respuesta de la planta a las adiciones de nutrientes depende de la especie y la forma en que se aplique el fertilizante.

\section{CONCLUSIONES}

El repollo corazón de buey mostró mejor respuesta productiva a la combinación de guano de isla y abono foliar, logrando incrementar hasta en un 57,2\%. Sin embargo entre los tratamientos también destacan los que solo consideraban guano de isla o sólo humus de lombriz, que de igual forma superaron al testigo absoluto. Esto sugiere que en una eventual situación de no disposición de abonos foliares, una buena opción es la aplicación de enmiendas para incrementar el rendimiento del cultivo.

La adición de abonos foliares a los planes de fertilización, según se observó en el presente trabajo, mejoran los rendimientos del cultivo; sin embargo hay que tener en cuenta que su incorporación genera mayores gastos e incrementa los costos de producción, por lo tanto es necesario hacer un análisis de costo beneficio previo. 


\section{REFERENCIAS BIBLIOGRÁFICAS}

Alshaal, T. \& H. El-Ramad. 2017. "Foliar application: from plant nutrition to Biofortification". Env. Biodiv. Soil Security 1: 71-83.

Bellido, C. 2010. Efecto del guano de islas incubado con solución de microorganismos en el crecimiento del tomate (Lycopersicon esculentum Mill) en Ayacucho a 2750 msnm. Tesis de Grado. Facultad de Ciencias Agrarias. UNSCH. Ayacucho (Perú).

Fernández, V., T. Sotiropoulos \& P. Brown. 2015. Fertilización foliar: Principios científicos y práctica de campo. Asociación internacional de la industria de los fertilizantes. París (Francia): Asociación Internacional de la Industria de Fertilizantes (IFA).

FHIA - Fundación Hondureña de Investigación Agrícola. 2012. Repollo de invierno: alternativa para diversificar la producción en el valle de Comayagua. Comayagua (Honduras): FHIA.

Guerrero, R. 1998. Fertilización de cultivos en clima frío.Caracas (Venezuela): Monómeros Colombo Venezolanos.

Kannan, L., R. Liyanage \& J. R. Lay. 2009 "Evaluation of beta defensin 2 production by chicken heterophils using direct MALDI mass spectrometry". Mol Immunol 46 (15):3151-3156.

Kuepper, G. 2003. Foliar fertilization. Naironi (Kenia): ATTRA.

Matus, M. 1999. Evaluación de tres distancias de siembra y dos dosis de fertilización en el desarrollo, rendimiento y rentabilidad del cultivo de repollo (Brassica oleraceae L.) El Tisey. Estelí. Tesis de Grado. Universidad Nacional Agraria. Managua (Nicaragua).

Molina, E. 2002. "Fuentes de fertilizantes foliares". Fertilización foliar: principios y aplicaciones $1: 26-35$

Morales, M. 2010. Evaluación de la fertilización foliar orgánica e inorgánica en el cultivo de repollo (Brassica oleracea L. var. Capitata). Tesis de Grado. Universidad Autónoma Agraria Antonio Narro. Saltillo (México).

Nina, O. 2014. Efecto del abonamiento con dos tipos de preparación de compost en el rendimiento de cuatro variedades de repollo (Brassica oleracea L. var. capitata) en K'yara-Cusco. Tesis de Grado. Universidad Nacional de San Antonio Abad del Cusco. Cusco (Perú).

Rotondo, R., I. Firpo, L. Ferreras, S. Toresani, S. Fernández \& E. Gómez. 2009. "Efecto de la aplicación de enmiendas orgánicas y fertilizante nitrogenado sobre propiedades edáficas y productividad en cultivos hortícolas". Horticultura Argentina 28 (66): 31-44.

Salas, R. 2002. "Herramientas de diagnóstico para definir recomendaciones de fertilización foliar”. Fertilización foliar: principios y aplicaciones 1:7-18.

Tejada, M. \& J. Gonzalez. 2006. "Crushed cotton gin compost on soil biological properties and rice yield". European Journal of Agronomy 25: 22-29.

Vargas, C., E. Suárez, J. López \& J. Moreno. 2008. "Influence of Compost Amendment on Soil Biological Properties and Plants". Dynamic Soil, Dynamic Plant. 2: 21-29.

Zedelón, O., G. Chavarría \& R. García. 2014. Efecto de tres métodos de fertilización orgánica en la producción de repollo (Brassica olerácea). Tesis de Grado. Universidad Autónoma de Nicaragua. León (Nicaragua). 